\title{
Use of the Gabor Filter for Edge Detection in the Analysis of Zinc Oxide Nanowire Images
}

\author{
B. E. Scanley ${ }^{1}$, T. E. Sadowski ${ }^{1}$, C. I. Pelligra ${ }^{2}$, M. E. Kreider ${ }^{3}$, C.O. Osuji ${ }^{2,4}$ and C. C. Broadbridge ${ }^{1,4}$ \\ ${ }^{1}$ Department of Physics, Southern Connecticut State University, New Haven, U.S.A. \\ ${ }^{2}$ School of Engineering and Applied Science, Yale University, New Haven, U.S.A. \\ ${ }^{3}$ CRISP Research Experiences for Undergraduates (REU) Program at Yale/SCSU, New Haven, U.S.A. \\ ${ }^{4}$ Center for Research on Interface Structures and Phenomena at Yale/SCSU, New Haven, U.S.A.
}

Semi-automated processing of microscopy images can significantly enhance the capacity for objective nanostructure characterization. However, background complexity, intricate object features and overlapping of objects can make this a difficult task. Here we describe use of the Gabor filter $[1,2]$ to facilitate the identification and outlines of the top surfaces of nanowires of zinc oxide ( $\mathrm{ZnO})$, imaged with scanning electron microscopy (SEM). The Gabor filter is a sinusoidal function multiplied by a Gaussian envelope. The sinusoidal shape makes the filter sensitive to spatial frequencies and the Gaussian envelope limits the frequency sensitivity to localized areas of the image. In particular, the Gabor filter enhances lines and edges in a direction perpendicular to the direction of the sinusoid. This sensitivity to linear parts of an image make it particularly well suited to analysis of the top surfaces of the $\mathrm{ZnO}$ nanowires which have a regular hexagonal shape and bright edges in the SEM images.

$\mathrm{ZnO}$ nanowire forests have potential uses in low-cost, high-efficiency hybrid photovoltaics [3]. For this particular application, optimally the nanowire diameters would be small $(10-30 \mathrm{~nm})$ with a nanowire separation of twice the exciton diffusion length $(15-30 \mathrm{~nm})$. SEM imaging was done to assess the fabrication process as synthesis conditions were optimized. The fabrication involved several steps. A silicon substrate was spin coated with a thin layer of zinc-acetate which was thermally decomposed in air to a seed-layer of $\mathrm{ZnO}$. The $\mathrm{ZnO}$ seed layer was spin coated with a layer of lamellar-forming polystyrene-poly(methyl methacrylate), (PS-PMMA) block co-polymer. Under the right annealing conditions, domains of the PMMA extend from the sample surface through to the underlying seeded substrate. The PMMA part of the PS-PMMA block co-polymer is then etched away using UV light leaving a template with evenly spaced small pores that extend to the $\mathrm{ZnO}$ seed layer. The template is immersed in a zinc containing hydrothermal solution and heated while the $\mathrm{ZnO}$ nanowires crystallize. Scanning electron microscope images of zinc oxide nanowires were obtained with a Zeiss Sigma VP SEM. ZnO nanowires were coated with a 4-6 nm gold layer in order to prevent charging artifacts.

Images were processed in Matlab using a modification of publically available Gabor Filter plugin (http://www.mathworks.com/matlabcentral/fileexchange/23253-gabor-filter). Images were filtered at 40 angles with each pixel in the output image containing the maximum intensity of the 40 filtered images. The filtered image was smoothed with a Gaussian kernel. Inner boundaries of objects were found using a Matlab implementation of a Moore-Neighbor tracing algorithm.

Figure 1 shows an example of an image processed with this algorithm. The image chosen for analysis had a very uneven distribution of nanowires and an irregular background but fairly bright nanowire edges. The original image is in grayscale and the top surfaces of the $\mathrm{ZnO}$ nanowires are outlined in red. A majority of the nanowires were identified and there were only a few instances of misidentification. Nanowires that were on edge of the image would not have been found by the boundary algorithm. Some 
of the nanowires that had lower edge contrast were missed. Misidentification of regions occurred particularly on the sides of the nanowires and in-between close groupings of nanowires.

The Gabor filter appears to be a promising strategy to facilitate analysis of these types of images. Several modifications of this method might improve the feature detection such as iterative filtering, linear image morphing, use of thresholding and brightness, contrast windowing, and post-processing of the images with the circular Hough Transform. Pre-processing techniques might enable the use of edge detection algorithms such as the Canny and Sobel methods. SEM imaging parameters such as the accelerating voltage and choice of detectors might be modified to enhance edge effects. The authors acknowledge support of this research [4].

[1] D. J. Gabor, J. Instn. Elect. Engrs 93 (1946), p. 429.

[2] R. Mehrotra, K. R. Namuduri, and N. Ranganathan, Pattern Recognition 25 (1992), p. 1479.

[3] S. Zhang, C. I. Pelligra, G. Keskar, J. Jiang, P. W. Majewski, A. D. Taylor, S. Ismail-Beigi, L. D.

Pfefferle, and C. O. Osuji, Adv. Materials 24 (2012), p. 82.

[4] The authors acknowledge primary financial support from CRISP and the CRISP Research

Experiences for Undergraduates (REU) program (NSF MRSEC DMR 1119826). Partial support by the

DOE (DE-SC0005072).

${ }^{*}$ M. Kreider is currently at the School of Engineering, Massachusetts Institute of Technology, Cambridge, U.S.A.

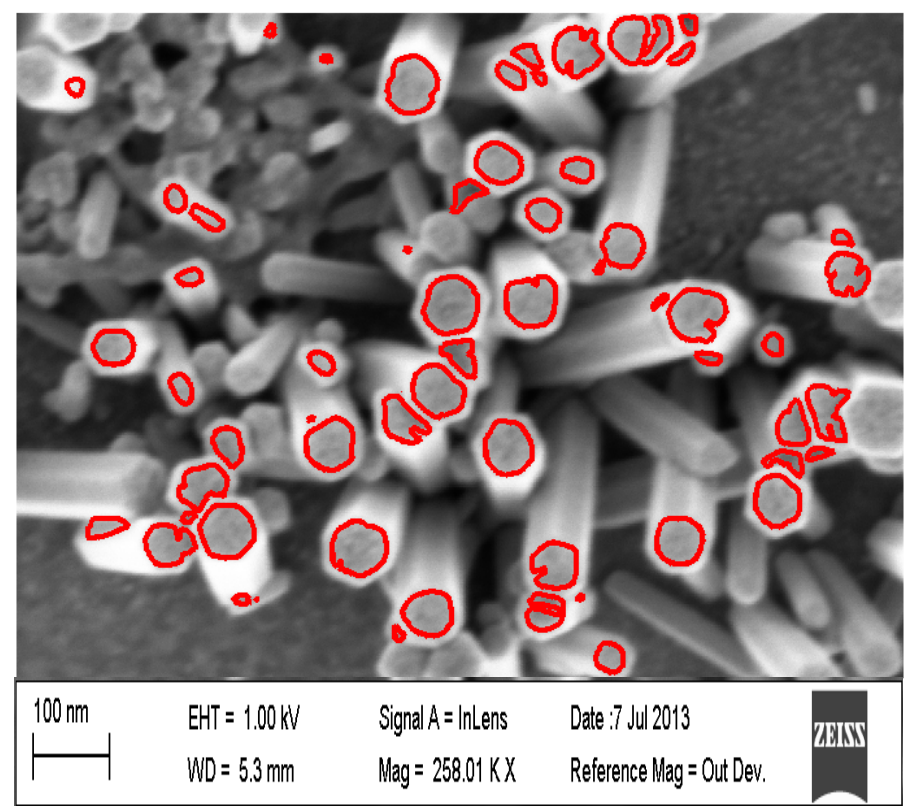

Figure 1. SEM image of zinc oxide nanowires with superimposed boundaries of nanowires. Boundaries were determined using a Gabor filter and Gaussian smoothing followed by a boundary detection algorithm. The SEM image was obtained using an accelerating voltage of $1 \mathrm{kV}$, working distance of 5.3 $\mathrm{mm}$, an InLens detector and a magnification of $258 \mathrm{kX}$. 